\title{
Kadsuphilactones A and B, Two New Triterpene Dilactones from Kadsura philippinensis
}

\author{
Ya-Ching Shen, ${ }^{*}{ }^{\dagger}$ Yu-Chi Lin, ${ }^{\dagger}$ Michael Y. Chiang, ${ }^{*}$ \\ Sheau Farn Yeh, ${ }^{\S}$ Yuan-Bin Cheng ${ }^{\dagger}$ and Chia-Ching Liao ${ }^{\dagger}$ \\ ${ }^{\dagger}$ Department of Marine Resources, National Sun Yat-Sen University, \\ Kaohsiung 804, Taiwan, Republic of China \\ ${ }^{\ddagger}$ Department of Chemistry, National Sun Yat-Sen University, Taiwan, Republic of China \\ $\S$ Institute of Biochemistry, National Yang-Ming University, Taipei, Taiwan, Republic of China

\section{List of Supporting Information}

1. ${ }^{1} \mathrm{H}$ NMR ${ }^{13} \mathrm{C}$ NMR spectra and X-ray crystallographic data of compound 1

2. ${ }^{1} \mathrm{H} N M R{ }^{13} \mathrm{C}$ NMR spectra and X-ray crystallographic data of compound 2 


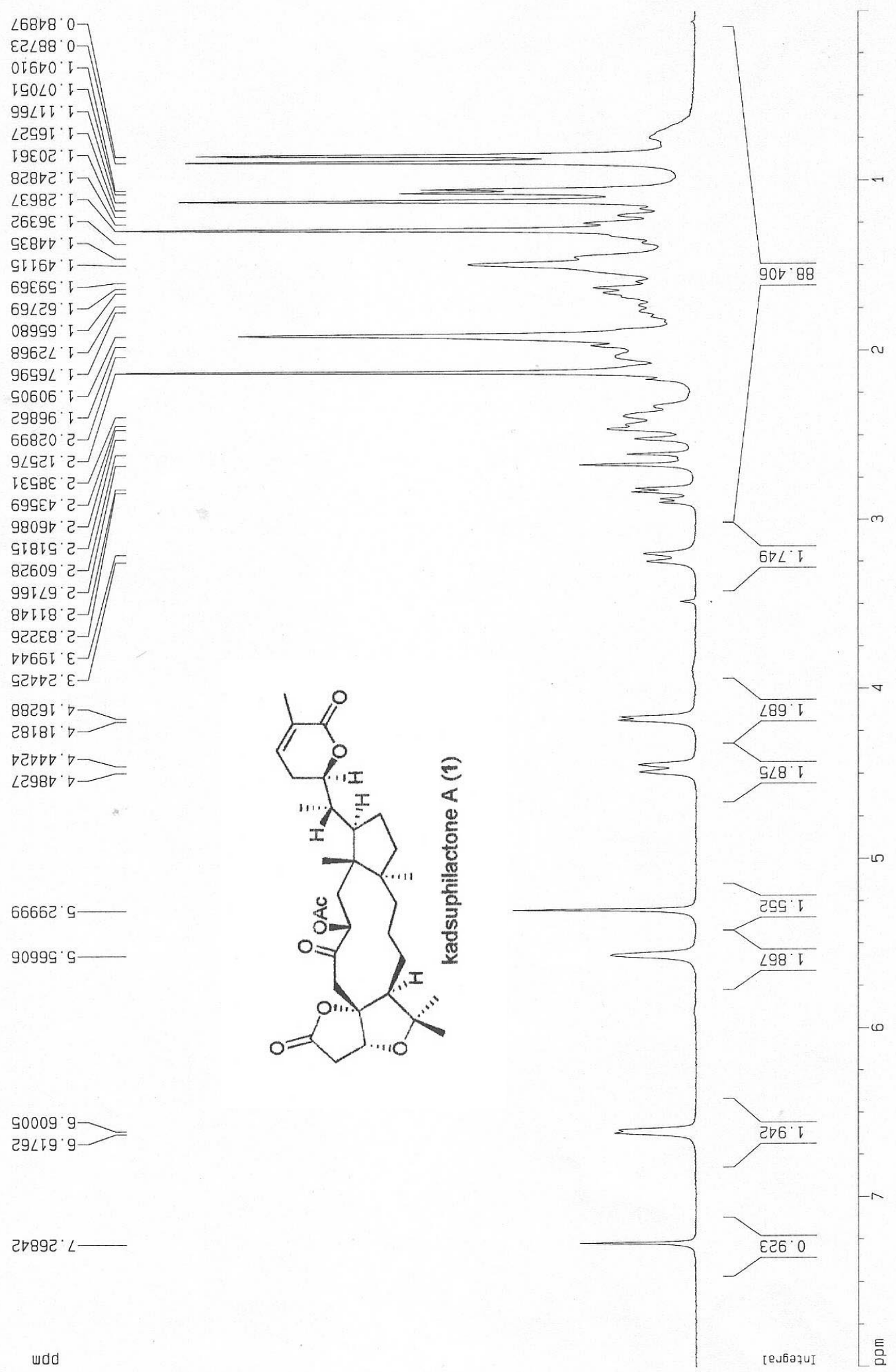


S3

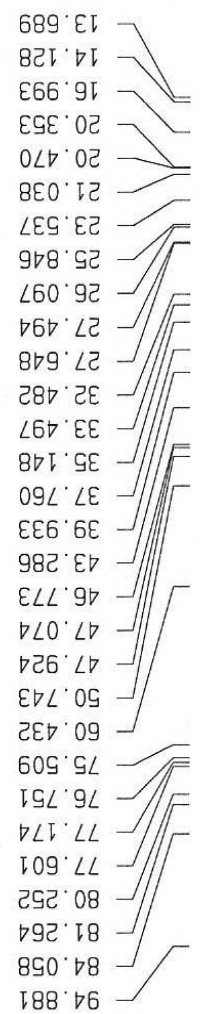

6โว $82 Г-$

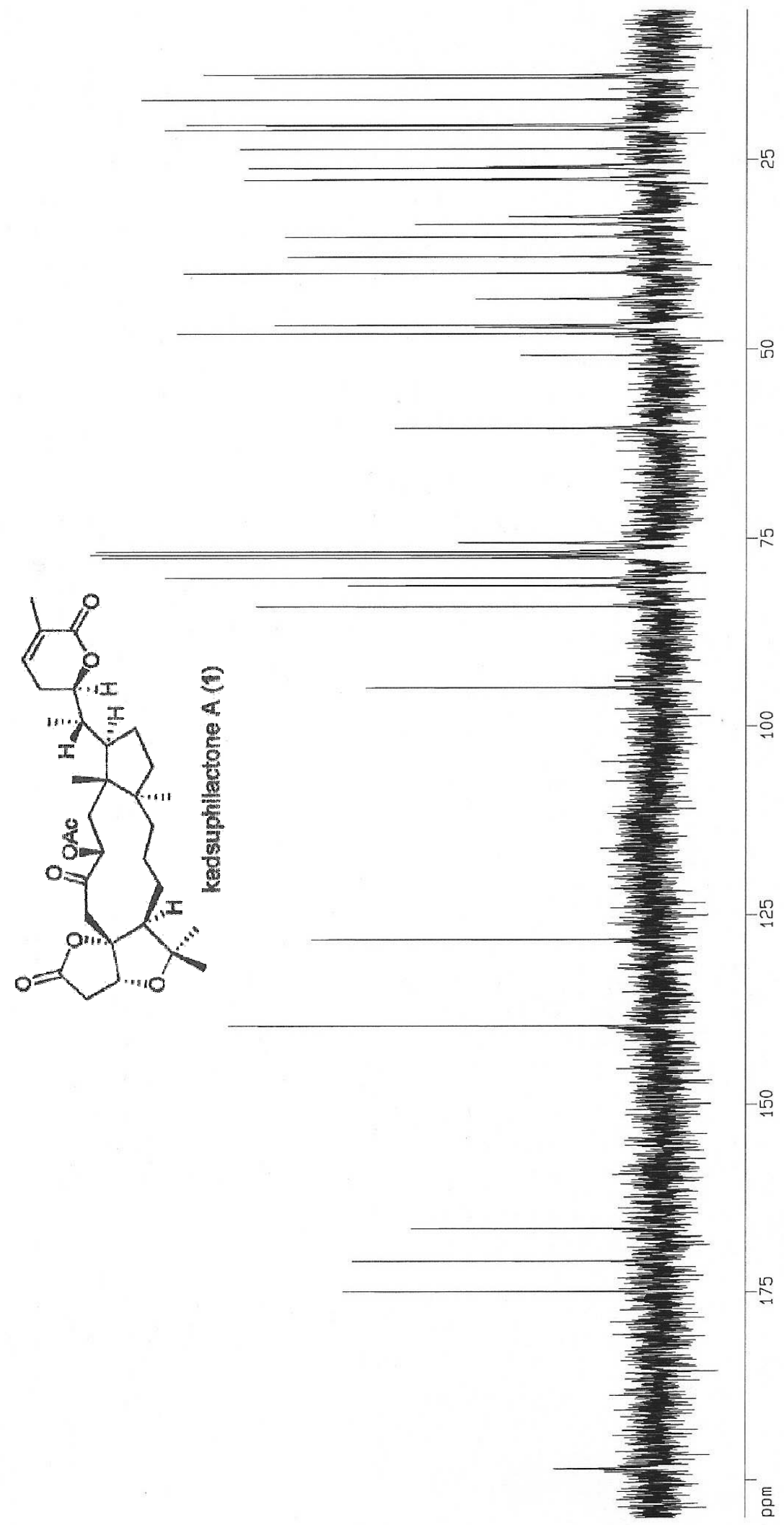

IGG ' 991-

$298^{\circ} 0 \angle 1$

$088^{\circ} \forall \angle I$

8โ†.86โ 


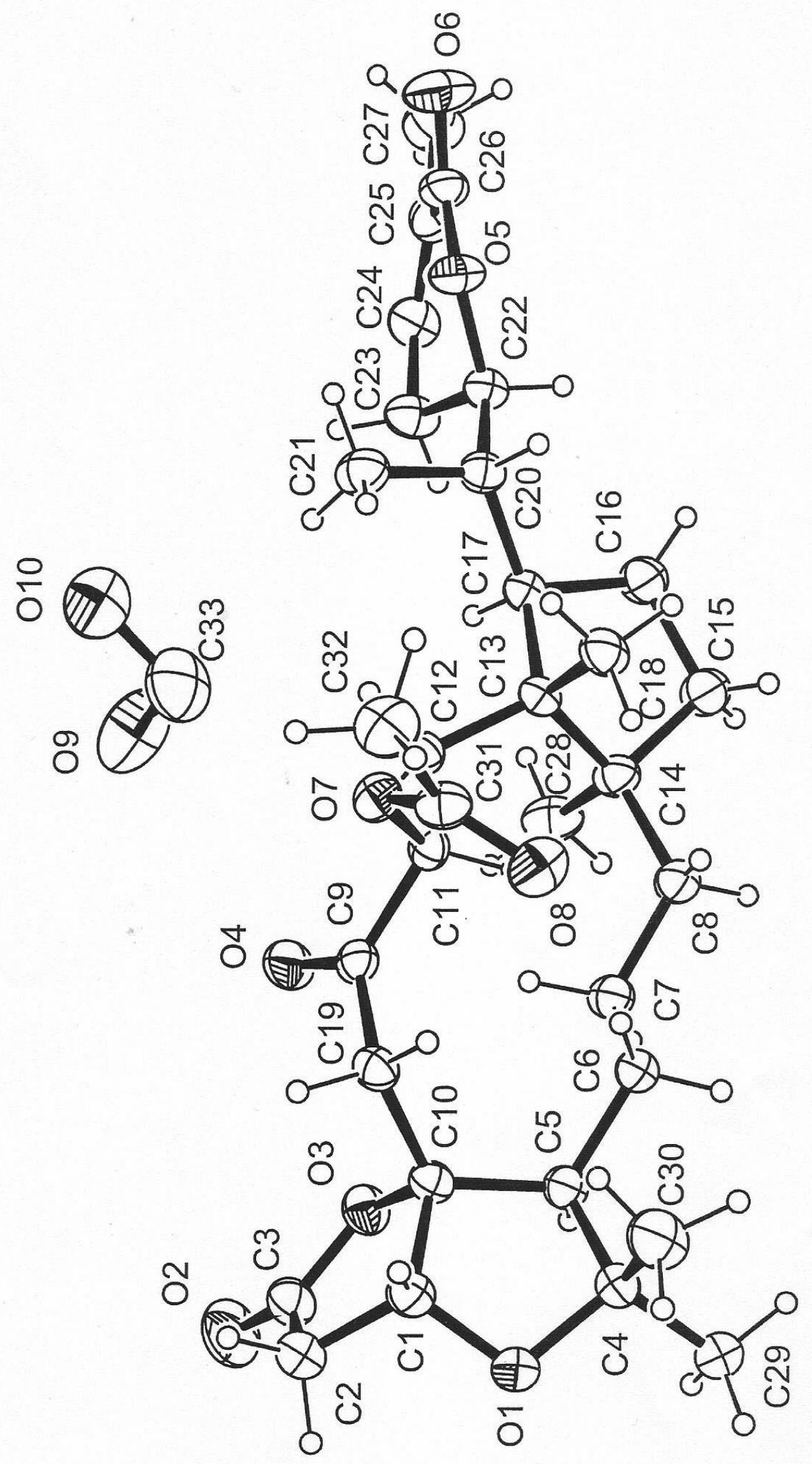

S4 
Table 1. Crystal data and structure refinement for kadsuphilactone A

Identification code

Empirical formula

Formula weight

Temperature

Wavelength

Crystal system, space group

Unit cell dimensions

Volume

Z, Calculated density

Absorption coefficient

$\mathbf{F}(\mathbf{0 0 0})$

Crystal size

Theta range for data collection

Limiting indices

Reflections collected / unique

Completeness to theta $=\mathbf{2 5 . 9 8}$

Absorption correction

Refinement method kadsuphilactone A

\section{C33 H50 O9}

$\mathbf{5 9 0 . 7 3}$

293(2) K

0.71069 A

Trigonal, P31 (No. 144)

$$
\begin{aligned}
& a=b=10.4666(14) A \quad \text { alpha }=90 \mathrm{deg} . \\
& \text { beta }=90 \mathrm{deg} \text {. } \\
& c=25.264(6) A \\
& \text { gamma }=120 \mathrm{deg} \text {. }
\end{aligned}
$$

2396.8(6) $A^{\wedge} 3$

3, $\quad 1.228 \mathrm{Mg} / \mathrm{m}^{\wedge} 3$

$0.088 \mathrm{~mm}^{\wedge}-1$

960

$0.8 \times 0.6$ x $0.2 \mathrm{~mm}$

2.25 to $25.98 \mathrm{deg}$.

$-11<=\mathrm{h}<=11,0<=\mathrm{k}<=12,0<=\mathrm{l}<=31$

$3473 / 3208[R($ int $)=0.0209]$

$99.9 \%$

psi-scan

Full-matrix least-squares on $\mathrm{F}^{\wedge} 2$ 
Data / restraints / parameters

Goodness-of-fit on $\mathrm{F}^{\wedge} 2$

Final $R$ indices [I $>2 \operatorname{sigma}(\mathrm{I})]$

$\mathbf{R}$ indices (all data)

Absolute structure parameter

Largest diff. peak and hole
3208 / 1 / 395

1.047

$\mathrm{R} 1=\mathbf{0 . 0 4 2 6}, \mathrm{wR2}=\mathbf{0 . 1 1 2 7}$

$\mathrm{R} 1=\mathbf{0 . 0 7 1 2}, \mathrm{wR} 2=\mathbf{0 . 1 3 0 5}$

$-0.4(14)$

0.278 and -0.241 e. $A^{\wedge}-3$ 


\section{S7}

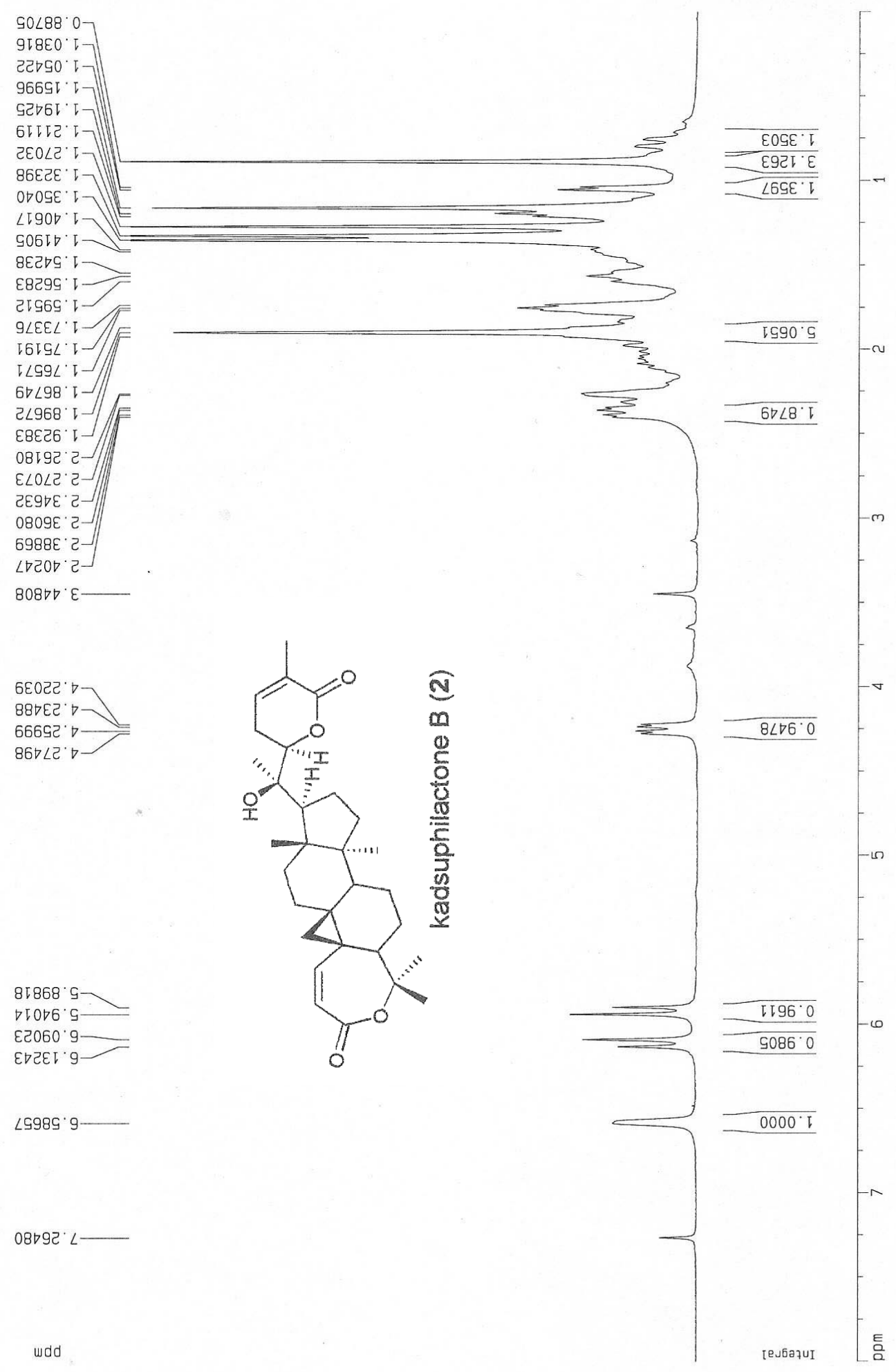


S8

$\nabla 68.91-$

$66 \mathrm{~T} \cdot 6 \mathrm{I}$

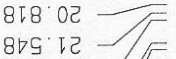

6हा.55

टा厂・呵

$\varepsilon 89^{\circ} \nabla 2-1$

$609.95]$

Lटจ 85

$\nabla 00.62]$

$602 \cdot 62$

$660^{\circ} \varepsilon \varepsilon$

นโ $\varepsilon \varepsilon$

$\left.\varepsilon 59^{\circ} \nabla \varepsilon\right]$

8I6. $\nabla \nabla$

$\angle 9 l \cdot 97$

$68 \nabla \cdot 9 \nabla]$

SI6. 80

969.09
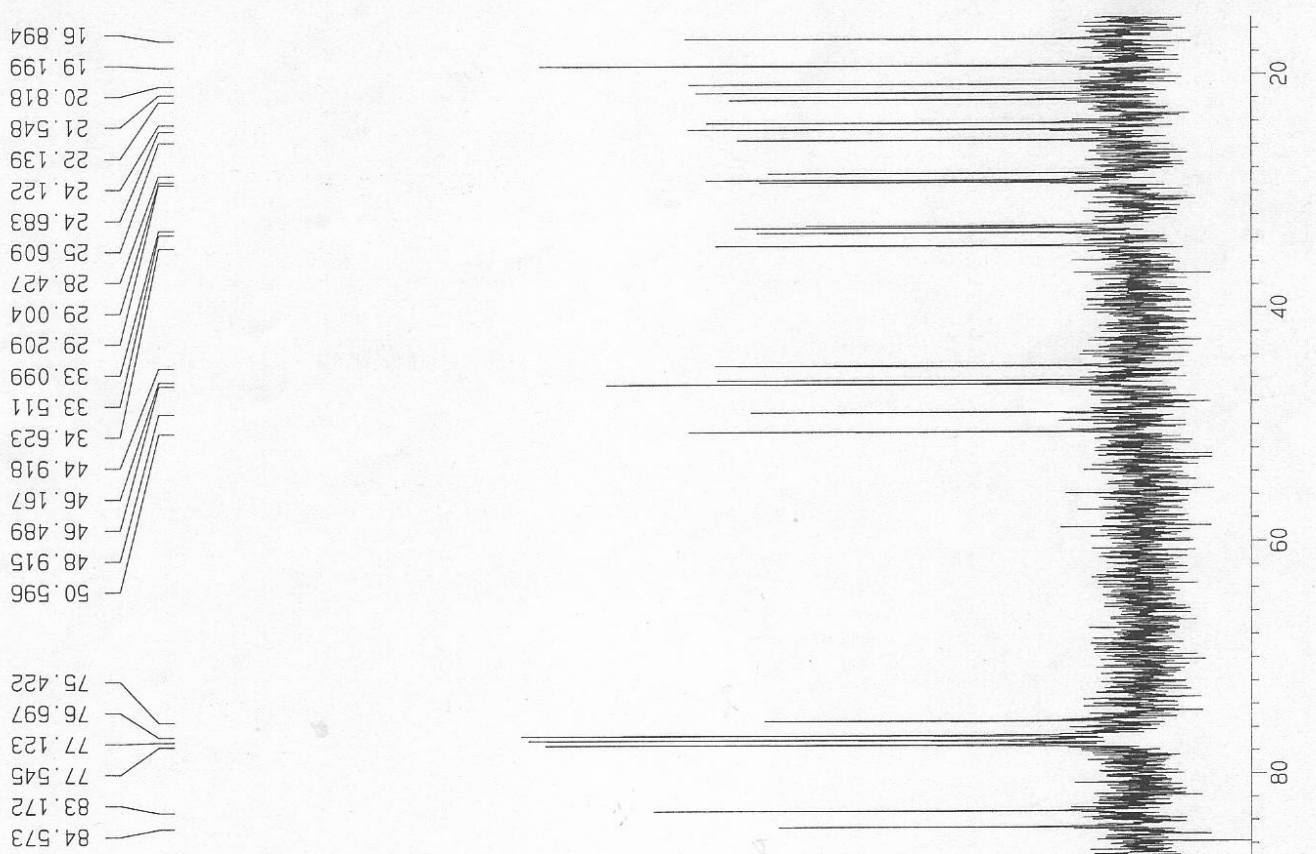

962 0टा

O७E 8टレ

$\nabla 20.6 \varepsilon \downarrow-$

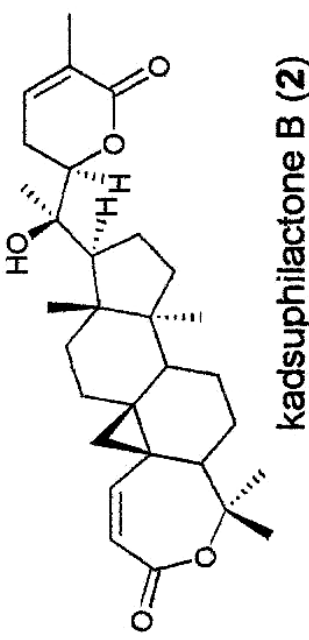

IILOST -

067.991 -

$\angle 2 D^{\circ} \angle 91-$

욱

ஐ

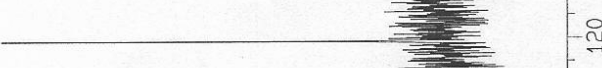

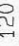

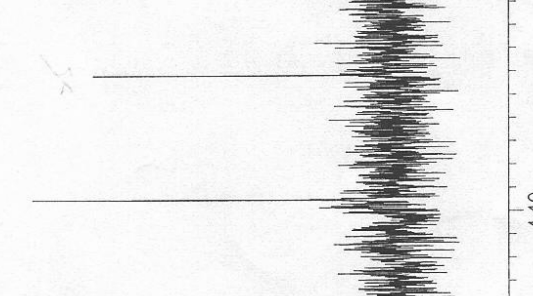

웜
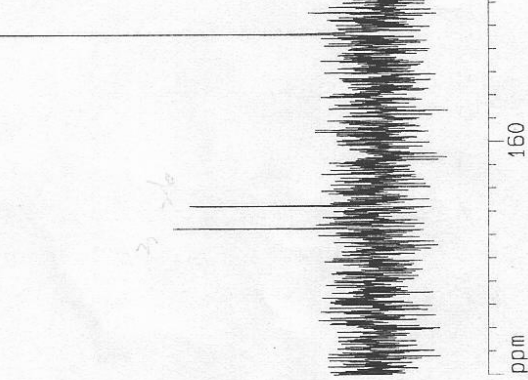


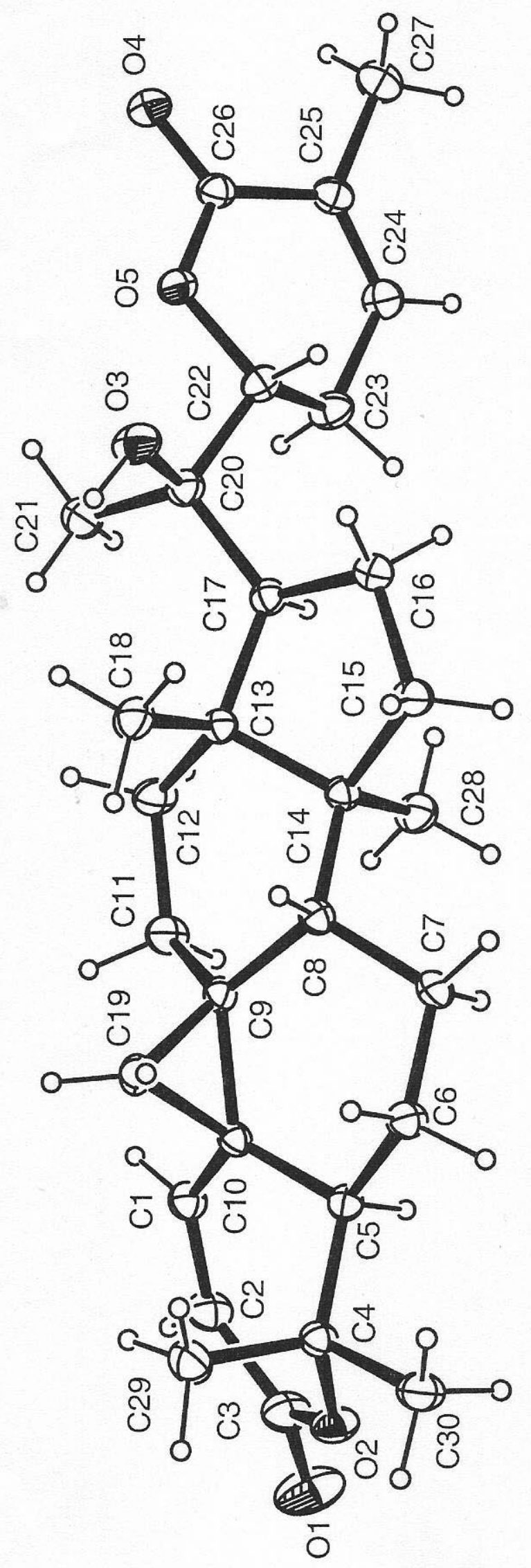

S9 
Table 2. Crystal data and structure refinement for kadsuphilactone $\mathbf{B}$.

Identification code

Empirical formula

Formula weight

Temperature

Wavelength

Crystal system, space group

Unit cell dimensions

Volume

Z, Calculated density

Absorption coefficient

$\mathbf{F}(000)$ kadsuphilactone B

C30 H43 O5.50

491.64

293(2) K

0.71069 A

Monoclinic, P21 (No. 4)

$$
\begin{gathered}
a=15.305(2) \mathrm{A} \quad \text { alpha }=90 \mathrm{deg} . \\
\mathrm{b}=11.735(3) \mathrm{A} \quad \text { beta }=106.94(1) \mathrm{deg} . \\
\mathrm{c}=15.339(3) \mathrm{A} \quad \text { gamma }=90 \mathrm{deg} .
\end{gathered}
$$

$$
2635.5(10) A^{\wedge} 3
$$

4, $1.239 \mathrm{Mg} / \mathrm{m}^{\wedge} 3$

$0.084 \mathrm{~mm}^{\wedge}-1$

1068 
Crystal size

Theta range for data collection 2.22 to $26.01 \mathrm{deg}$.

Limiting indices

Reflections collected / unique

Completeness to theta $=\mathbf{2 6 . 0 1}$

Absorption correction

Refinement method

Data / restraints / parameters

Goodness-of-fit on $\mathrm{F}^{\wedge} 2$

Final $R$ indices [I $>2 \operatorname{sigma}(\mathrm{I})]$

$\mathbf{R}$ indices (all data)

Absolute structure parameter

Largest diff. peak and hole $0<=h<=18,0<=k<=14,-18<=l<=18$

$0.5 \times 0.5 \times 0.2 \mathrm{~mm}$

S11

$5648 / 5441[R($ int $)=0.0170]$

$99.9 \%$

psi-scan

Full-matrix least-squares on $\mathrm{F}^{\wedge} \mathbf{2}$

5441 / 1 / 635

0.998

$\mathrm{R} 1=0.0440, w R 2=0.1168$

$\mathrm{R} 1=0.0926, \mathrm{wR} 2=0.1418$

$-1.2(14)$

0.581 and -0.224 e. $A^{\wedge}-3$ 